\title{
$\rightarrow$ \\ Impedancia intraluminal multicanal asociada a pHmetría en el estudio de la enfermedad por reflujo gastroesofágico
}

En el artículo de de la Morena y cols. (1) se describen algunas de las aportaciones que la impedancia intraluminal multicanal asociada a la pHmetría (IIM-pH) hace al estudio de la enfermedad por reflujo gastroesofágico (ERGE), llegando a considerar a la IIM-pH como el patrón oro en el estudio de la ERGE. En este editorial describimos algunas de las limitaciones de esta técnica que reducen su utilidad y nos obligan a ser "prudentes" en su valoración.

En medicina, los métodos de estudio basados en la medida de la impedancia han sido utilizados desde hace tiempo con el fin de disponer de una monitorización no invasiva de diferentes órganos. Esta técnica se basa en la medida de los cambios de la resistencia al paso de una corriente eléctrica entre dos electrodos que surgen como consecuencia de modificaciones funcionales o estructurales del órgano en estudio. En gastroenterología, algunas de sus aplicaciones iniciales fueron la monitorización de las contracciones intestinales $(2)$ y el estudio del vaciado gástrico $(3,4)$.

Los primeros estudios de la fisiopatología esofágica con esta técnica se realizaron hace más de quince años por Silny (5). Desde entonces, se han logrado grandes progresos, tanto en el desarrollo de los equipos como en el reconocimiento de los diferentes patrones de registro.

En los procedimientos actuales se utiliza un fino catéter (de unos $2 \mathrm{~mm}$ de diámetro) que contiene varios pares de electrodos en forma de anillos que, colocados en su superficie, permiten la toma de datos a diferentes niveles. La distancia entre los electrodos de cada par es de $2 \mathrm{~cm}$ y la separación entre cada par varía dependiendo de la longitud del esófago objeto del estudio (niños o adultos). Una débil corriente eléctrica es aplicada a uno de los electrodos y se registra la resistencia al flujo de dicha corriente hacia el otro. Los cambios en la impedancia intraesofágica, determinada por la medición de la conductividad eléctrica (impedancia intraluminal multicanal -IIM-) permiten la identificación de diferentes eventos intraluminales, incluyendo la composición del contenido intraesofágico (aire, líquido o mixto) y el sentido en el que este progresa, tránsito anterógrado del material deglutido o paso retrógrado de material refluido.

La información aportada por la IIM es, por tanto, complementaria a la de la manometría y la pHmetría. Es por eso por lo que generalmente se realizan estudios combinados con estas técnicas. Cuando se utiliza de forma conjunta con la manometría se obtiene información de la actividad contráctil y del movimiento del bolo dentro del esófago. Combinada con la pHmetría permite detectar la composición, distribución y aclaramiento del reflujo, diferenciando entre reflujo líquido (independientemente del pH ácido o no ácido), gas y combinado de gas y líquido (6).

El análisis de todos estos factores con los datos de la pHmetría ha llevado a algunos autores a distinguir hasta 11 tipos diferentes de episodios de reflujo (7) que ge- 
neralmente se suelen reducir a tres: episodios ácidos $(\mathrm{pH}<4)$, débilmente ácidos $(\mathrm{pH}>4 \mathrm{a}<7)$ y no ácidos ( sin cambios apreciables en el pH).

Es evidente que la introducción de la IIM-pH aporta nuevas posibilidades en el estudio de la ERGE. Constituye el método más exacto y detallado para detectar el reflujo de cualquier tipo (8). Sin embargo, los estudios de IIM tienen aún importantes limitaciones que se deben tener en cuenta a la hora de su indicación y análisis.

En cuanto a las indicaciones es importante señalar que, aunque existen marcadas diferencias en el valor absoluto de la impedancia en función de la presencia de contenido intraesofágico y de si este es líquido o gas, en esta técnica lo que se valora son los cambios de la línea base, de tal manera que en pacientes con alteraciones histológicas de la mucosa esofágica (esofagitis grave, esófago de Barrett) y/o con alteraciones graves de la motilidad que interfieren el tránsito o el aclaramiento esofágico y que por tanto ocasionan retención de líquidos en el esófago, la impedancia de referencia es muy baja, con una línea base casi plana. Esto dificulta el análisis de la IIM-pH y hace, en algunos casos, casi imposible interpretar el estudio (9).

Por otra parte, los estudios con IIM-pH en pacientes sin tratamiento han demostrado que aproximadamente una tercera parte de todos los episodios de reflujo son débilmente ácidos (10), y que estos también pueden producir pirosis y regurgitación (11). Sin embargo, el reflujo débilmente ácido en los pacientes que no consumen inhibidores de secreción ácida sólo parece responsable de una minoría de síntomas (12). Por tanto, en este grupo de enfermos en los que algunos estudios señalan que la IIM-pH permite identificar una proporción considerablemente más alta de pacientes con síntomas inducidos por reflujo (7), no está suficientemente demostrado que su impacto y su relación coste/beneficio sean realmente favorables.

Esta técnica está particularmente indicada para la detección y cuantificación del RGE en: a) pacientes en tratamiento con inhibidores de la secreción ácida en los que persisten los síntomas; b) pacientes en los que la sintomatología aparece principalmente en el periodo postprandial y/o con ingesta muy frecuente de comidas, es decir, niños recién nacidos, lactantes, etc.; c) enfermos con síntomas laringofaríngeos o respiratorios (microaspiración) $(13,14)$; y d) pacientes con escasa o nula capacidad de producir ácido (gastritis atrófica o tras gastrectomía). En este último apartado se debe señalar que el valor de esta técnica cuando se sospecha la existencia de reflujo biliar está por determinar. Así, los escasos estudios realizados simultáneamente con Bilitec ${ }^{\circledR}$ indican que el reflujo no ácido (tal como se define con la IIM$\mathrm{pH}$ ) y el reflujo biliar (tal como se define en los registros mediante espectrofotometría con Bilitec $\left.{ }^{\circledR}\right)$ no son equiparables y, por tanto, se requieren ambos medios de diagnóstico para hacer un estudio correcto (15).

En cuanto a las limitaciones relacionadas con el análisis, el primer aspecto a considerar es que el software de análisis automático no es suficientemente fiable y generalmente sobreestima el número de eventos de reflujo no-ácido (16-18). Es por tanto imprescindible el análisis visual adicional realizado por personal con experiencia para evitar falsos-positivos, lo que inevitablemente conlleva un componente subjetivo (19) y aumento de costes.

Uno de los puntos fuertes de la IIM es la mayor capacidad de esta técnica para establecer la posible existencia de asociación entre síntomas y reflujo. Para poder establecer esta asociación, uno de los primeros pasos a realizar es determinar si cada episodio de reflujo es sintomático o no. Se consideran episodios sintomáticos aquellos en los que el síntoma se produce dentro de un periodo determinado de tiempo desde el principio del episodio de reflujo. Es aquí donde surge uno de los primeros problemas, ¿qué intervalo de tiempo es el más adecuado? Mientras algunos autores 
consideran que es de 2 minutos $(7,20)$, otros opinan que debería ser de 5 minutos (21). Es evidente que cuanto mayor sea el tiempo más posibilidades existen de poder relacionar síntomas con episodios de reflujo.

Por último, no está bien determinado qué fórmula matemática es la más correcta para poder precisar la posible existencia de relación entre síntomas y reflujo. Las tres más utilizadas son: el índice de síntomas (IS), el índice de sensibilidad de los síntomas (ISS) y la probabilidad de asociación de síntomas (PAS) $(7,22)$. Todas ellas tienen ventajas e inconvenientes que las alejan del índice ideal (22). El IS es el más utilizado; su defecto principal es que no tiene en cuenta el número total de episodios de reflujo. Cuanto mayor sea el número de eventos considerados como episodios de reflujo, más alta es la posibilidad de que un síntoma se produzca por casualidad en el periodo de los 2-5 minutos posteriores al mismo. De igual manera, el ISS no tiene en cuenta el número de episodios de síntomas, y un elevado número de episodios sintomáticos produce probablemente un índice alto. La ventaja del PAS es que tiene en cuenta todos los factores relevantes; sin embargo, su desventaja es que este parámetro es difícil de calcular manualmente y en opinión de algunos autores tiene un valor estadístico arbitrario (22).

Sin duda, una de las principales aportaciones de la IIM-pH es la de poder demostrar la ausencia de relación entre síntomas y reflujo. Este aspecto de negatividad de relación es mucho más importante de lo que en principio se pudiera pensar, ya que en más de la mitad de los pacientes con síntomas persistentes a pesar de tratamiento se demuestra que el reflujo no es realmente el responsable de los mismos $(7,21)$, como también ocurre en el caso comentado por de la Morena y cols. en este número de la Revista Española de Enfermedades Digestivas (1). En este caso, la conclusión final no diefere de la que se tenía con la realización de pHmetría convencional, pero indudablemente la demostración de que los síntomas no tienen relación con los episodios de reflujo, con independencia del valor de $\mathrm{pH}$ que estos tengan, da mucha más seguridad para considerar el resultado realmente negativo y buscar un diagnóstico alternativo al RGE como causa de los síntomas.

Es cierto que la IIM asociada a manometría y/o a pHmetría contribuirá al mejor conocimiento de la fisiopatología esofágica y permitirá un mejor diagnóstico de nuestros pacientes. Estamos de acuerdo con Connor y Ritcher (22) al considerar que la situación actual de la técnica permite, con un alto nivel de probabilidad, rechazar el reflujo como causa de los síntomas, pero nos obliga a ser cautelosos en la valoración del significado clínico y la posible causalidad determinada por una puntuación positiva de la relación síntomas-episodios de reflujo.

La rápida difusión que este tipo de estudios está alcanzando en los últimos años será un fuerte estímulo para unificar criterios y definiciones entre los distintos grupos de investigación, obtener valores normales en grupos control amplios, realizar estudios de coste/beneficio, resolver las dificultades de interpretación y análisis de los registros y perfilar mejor las indicaciones, colocando a esta técnica en el lugar que le corresponde.

A. Ruiz de León San Juan y J. A. Pérez de la Serna Bueno 


\section{Bibliografía}

1. De la Morena F, Santander Vaquero C, Cantero J, Pérez T, Moreno Otero R. Impedancia esofágica intraluminal de canal múltiple: una nueva frontera en motilidad. Rev Esp Enferm Digest 2008; 100 (2): 86-9.

2. Darby CF, Hammond P, Taylor Morris IR. A method for the simultaneous detecttion o motility and myoelectrical activity in smooth muscle. Clin Phys Physiol Meas 1982; 3: 283-91.

3. McClelland GR, Sutton JA. Epigastric impedance: A non-invasive method for the assessment of gastric emptying and motility. Gut 1985; 26 (6): 607-14.

4. Sutton JA. Impedancia. En: Kumar D, Gustavson S, editores. Guía ilustrada sobre motilidad gastrointestinal. Ed. Esp. HaroFarma, S. A.; 1990. p. 115-9.

5. Silny J. Intraluminal multiple electric impedance procedure for measurement of gastrointestinal motility. J Gastrointest Motil 1991; 3: 151-62.

6. Hirano I. Review article: Modern technology in the diagnosis of gastro-oesophageal reflux disease -Bilitec, intraluminal impedance and Bravo capsule $\mathrm{pH}$ monitoring. Aliment Pharmacol Ther 2006; 23: 12-24.

7. Bredenoord AJ, Weusten BLAM, Timmer R, Conchillo JM, Smout AJPM. Addition of esophageal impedance monitoring to $\mathrm{pH}$ monitoring increases the yield of symptom association analysis in patients off PPI therapy. Am J Gastroenterol 2006: 101: 453-9.

8. Vaezi MF, Vela MF. The role of multichannel intraluminal impedance and $\mathrm{pH}$ monitoring in the diagnosis of gastroesophageal reflux disease. US Gastroenterol Rev 2007; 75-7.

9. Waśko-Czopnik D, Błoński W, Paradowski L. Diagnostic difficulties during combined multichannel intraluminal impedance and $\mathrm{pH}$ monitoring in patients with esophagitis or Barrett's esophagus. Advances in Medical Sciences 2007; 52: 196-8

10. Sifrim D, Holloway R, Silny J, Xin Z, Tack J, Lerut A, Janssens J. Acid, nonacid, and gas reflux in patients with gastroesophageal reflux disease during ambulatory 24-hour $\mathrm{pH}$-impedance recordings. Gastroenterol 2001: 120: 1588-98.

11. Vela MF, Camacho-Lobato L, Srinivasan R, Tutuian R, Katz PO, Castell DO. Simultaneous intraesophageal impedance and $\mathrm{pH}$ measurement of acid and nonacid gastroesophageal reflux: Effect of omeprazole. Gastroenterology 2001: 120: 1599-606.

12. Bredenoord AJ, Weusten BL, Curvers WL, Timmer R, Smout AJPM. Determinants of perception of heartburn and regurgitation. Gut 2006; 55: 313-8.

13. Ford CN. Evaluation and management of laryngopharyngeal reflux. JAMA 2005; 294: 1534-40.

14. Oelschlager BK, Quiroga E, Isch JA, Cuenca-Abente F. Gastroesophageal and pharyngeal reflux detection using impedance and 24-hour $\mathrm{pH}$ monitoring in asymptomatic subjects: Defining the normal environment. J Gastrointest Surg 2006; 10: 54-62.

15. Chwiesko A, Baniukiewicz A, Dabrowski A. Duodeno-gastro-esophageal reflux and Barrett's esophagus: Evaluation with esophageal multichannel intraluminal impedance and bilitec. Gut 2007; 56 (Supl. III): A197.

16. Roman S, Bruley des Varannes S, Pouderoux P, Chaput U, Mion F, Galmiche J, et al. Ambulatory 24 h esophageal impedance-pH recordings: Reliability of automatic analysis for gastro-esophageal reflux assessment. Gut 2006; 55 (Supl. V): A269.

17. Valitova ER, Bor S. The comparison of automatic vs. manual software analysis of a multichannel intraluminal impedance equipment. Gut 2007; 56 (Supl. III): A213.

18. Hemmink GJM, Aanen MC, Bredenoord AJ, Weusten BLAM, Smout AJPM. Automatic analysis of 24-h oesophageal impedance signals for detection of gastro-oesophageal reflux: How far are we? Gut 2007; 56 (Supl. III): A209.

19. López Alonso M, Moya MJ, Cabo JA, Ribas J, Macías MC, Silny J, et al. Reflujo gastroesofágico ácido y no ácido en el recién nacido. Datos preliminares de estudio con impedancia intraesofágica. Cir Pediatr 2005; 18: 121-6.

20. Lam HG, Breumelhof R, Roelofs JM, Van-Berge-Henewen, Smout AJ. What is the optimal time window in symptom analysis of 24-hour esophageal pressure and pH data? Dig Dis Sci 1994: 39: 402-9.

21. Mainie I, Tutuian, R, Shay, Vela M, Zhang X, Sifrim D, et al. Acid and non-acid reflux in patients with persistent symptoms despite acid suppressive therapy: A multicentre study using combined ambulatory impedance-pH monitoring. Gut 2006; 55: 1398-402.

22. Connor J, Richter J. El aumento del rendimiento también aumenta los resultados falsos positivos pero es más útil para excluir el RGE. Am J Gastroenterol (Ed. Esp.) 2006; 3: 162-5. 